\title{
RELAÇÕES BIOMÉTRICAS E AMBIENTAIS NO INCREMENTO DIAMÉTRICO DE Araucaria angustifolia NO PLANALTO SERRANO CATARINENSE
}

\author{
BIOMETRIC AND ENVIRONMENTAL RELATIONS IN THE DIAMETRIC INCREMENT OF Arau- \\ caria angustifolia IN SANTA CATARINA'S PLATEAU
}

\author{
Pollyni Ricken ${ }^{1}$ André Felipe Hess ${ }^{2}$ Geedre Adriano Borsoi²
}

\begin{abstract}
RESUMO
Este estudo analisa relações existentes entre o incremento médio anual em diâmetro e variáveis biométricas, morfométricas e ambientais em uma floresta nativa de Araucaria angustifolia no município de Lages-SC. Foram utilizados dados dendrocronológicos obtidos de 121 árvores dominantes e competidoras. Os indivíduos foram separados em 3 classes diamétricas, abrangendo indivíduos com DAP de $10 \mathrm{~cm}$ a $29,9 \mathrm{~cm}(\mathrm{C} 1)$, entre 30 e 49,9 $\mathrm{cm}(\mathrm{C} 2)$ e acima de $50 \mathrm{~cm}$ de DAP (C3). O banco de dados foi formado por uma matriz com as 121 árvores e 23 variáveis do ambiente, dendrométricas e morfométricas, que incluiu direcionamento de fluxo, acúmulo de fluxo, radiação solar, face de insolação, declividade, altitude, incremento médio em diâmetro, diâmetro, altura, distância (dominante/competidor), raios de copa por direção geográfica, área basal, idade, raio médio de copa, diâmetro de copa, área de copa, incremento em diâmetro por direção geográfica. Sendo que estas variáveis foram obtidas por meio dos algoritmos a partir do Modelo Digital de Elevação da área. As relações foram interpretadas com base nos resultados da análise multivariada, fazendo uso da análise de Cluster, análise fatorial e análise de componentes principais. As árvores apresentavam diâmetros variando entre 10 e $87,6 \mathrm{~cm}$ e alturas entre 5,3 e $21 \mathrm{~m}$, com incremento médio anual em diâmetro de 0,708 $\mathrm{cm}$, com média para 40 anos em análise retrospectiva. Constatou-se distinção de dois grupos e seis fatores diferenciados, que explicaram $81,5 \%$ da variância, sendo formados pelas características morfométricas e biométricas e pelas relações do incremento com as variáveis ambientais. As técnicas de análise multivariada podem ser aplicadas para auxílio na tomada de decisão no manejo florestal, pois comprovaram a correlação entre o incremento médio em diâmetro (essencial para compor o ciclo de corte florestal) e sua relação aos fatores do ambiente, índices de forma, espaço e dimensão dos indivíduos.

Palavras-chave: análise multivariada; manejo florestal; floresta ombrófila mista; fatores bioambientais.
\end{abstract}

\begin{abstract}
This study analyzes the relation between biometric, environmental and morphometric variables and the average annual increase in the diameter of a native forest of Araucaria angustifolia in Lages, SC state. To do so, we used dendrochronological data obtained from 121 dominant and competitor trees. The trees were separated into three diametrical classes, ranging from $10 \mathrm{~cm}$ to $29.9 \mathrm{~cm}(\mathrm{C} 1), 30$ to $49.9 \mathrm{~cm}(\mathrm{C} 2)$ and above $50 \mathrm{~cm}$ of DBH (C3). The database was formed by a matrix with 121 trees and 23 environmental, dendrometric and morphometric variables, which included flow direction, flow accumulation, solar radiation, face of insolation, slope, altitude, average diameter increment, diameter at breast height, height, distance (dominant/competitive), canopy rays in the north, south, east and west, basal area, age, average ray of canopy, canopy diameter, canopy area, average diameter increment by geographic direction. These variables were obtained using the algorithms from the Digital Elevation Model of the area. The relations were interpreted based on the results of multivariate analysis, using Cluster analysis, factor analysis and principal component

1 Engenheira Florestal, MSc., Doutoranda do Programa de Pós-Graduação em Engenharia Florestal da Universidade Federal do Paraná, Avenida Prefeito Lothário Meissner, 3400, Jardim Botânico, CEP 80210-170, Curitiba (PR), Brasil.pollyni7@hotmail.com

2 Engenheiro Florestal, Dr., Professor do Departamento de Engenharia Florestal, Universidade do Estado de Santa Catarina, Avenida Luiz de Camões, 2090, Conta Dinheiro, CEP 88520-000, Lages (SC), Brasil. andre.hess@ udesc.br / geedre.borsoi@udesc.br
\end{abstract}

Recebido para publicação em 15/02/2015 e aceito em 30/01/2018

Ci. Fl., v. 28, n. 4, out. - dez., 2018 
analysis. The trees had diameters ranging from 10 and $87.6 \mathrm{~cm}$ and heights between 5.3 and $21 \mathrm{~m}$, with average annual increment in diameter of $0.708 \mathrm{~cm}$, with an average of 40 in retrospective analysis. Together with the use of these techniques, we were able to distinguish two groups and six different factors that explain $81.5 \%$ of the variance, being formed by morphometric and biometric characteristics of the increment and the relationships with environmental variables. The techniques of multivariate analysis can be applied to aid the decision-making in forest management, because they proved the correlation between the average increment in diameter (essential to compose the forest cutting cycle) and its relation to environmental factors, index form, space and dimension of individuals.

Keywords: multivariate analysis; forest management; Araucaria forest; bioenvironmental factors.

\section{INTRODUÇÃO}

Na região Sul, encontra-se uma das mais importantes formações florestais do Brasil, a Floresta Ombrófila Mista. Tal denominação foi inicialmente utilizada por Veloso e Góes Filho (1982) e adotada pelo IBGE (1992) na classificação fisionômico-ecológica da vegetação brasileira. A concepção de Floresta Ombrófila Mista procede da ocorrência da mistura de floras de diferentes origens, definindo padrões fitofisionômicos típicos, em zonas climáticas caracteristicamente pluviais. Essa tipologia vegetal também é conhecida como Floresta com Araucária ou Mata de Araucária e antes de ser adequadamente conhecida, foi drasticamente reduzida a inexpressivos $10 \%$ de sua superfície original, em fragmentos alterados ou descaracterizados (LEITE; KLEIN, 1990).

As florestas são sistemas abertos que sofrem influência dos fatores ambientais, das características genéticas e da interação entre os indivíduos no espaço de ocupação. Sob esse aspecto os processos envolvidos na geração de modelos de crescimento e desenvolvimento de uma espécie e da comunidade não ocorrem apenas sob um fator, mas pelo conjunto de fatores, sendo relevante para o manejo a análise desse conjunto e não de aspectos isolados.

O crescimento das árvores depende dos fatores genéticos da espécie interagindo com o ambiente, compreendendo, desta forma, fatores climáticos: temperatura, precipitação, vento e insolação; solo: características físicas, químicas e biológicas; topografia: inclinação, altitude e exposição; competição: influência de outras árvores, vegetação rasteira e animais (FINGER, 2006).

Paula et al. (2004) descreveram que a distribuição diamétrica é uma das ferramentas utilizadas para a compreensão da sucessão. Segundo Siminski et al. (2004), é possível fazer uma avaliação prévia de condições dinâmicas da floresta através da distribuição diamétrica, permitindo assim previsões futuras quanto ao desenvolvimento da comunidade vegetal.

De acordo com Sanquetta et al. (2009), a projeção da estrutura diamétrica no tempo é de real importância para o manejo das florestas naturais, pois a partir dos diâmetros futuros pode-se estimar as produções e definir as intervenções que assegurem a sustentabilidade das mesmas. Além disso, tendo como base a estrutura diamétrica, é possível identificar nas classes aquela que apresenta deficit ou superavit de árvores.

Pretzsch (2014) constatou que a estrutura do dossel e morfologia das árvores afetam as condições ambientais dentro do povoamento e o crescimento das árvores, e por isso, muitas das funções e serviços da floresta.

Os parâmetros do manejo, crescimento-tempo, obtido com a dendrocronologia, fornecem informações da idade da árvore, dinâmica do uso do solo, impactos da mudança do ambiente e a associação ambiente-crescimento (SPEER, 2010).

A araucária produz anéis de crescimento sazonais e sua análise permite auxiliar o manejo florestal pelo estudo da relação do crescimento com as mudanças do ambiente e estrutura diamétrica da espécie. Cada anel de crescimento é formado como um calendário anual específico que permite analisar as relações entre o ambiente e o crescimento no tempo, podendo-se assim estimar como essa relação será no futuro sob um cenário de mudanças no ambiente (CATTANEO et al., 2013).

Tratando-se de manejo florestal se faz necessária a geração de modelos que predizem o crescimento, a produção e estrutura da floresta em uma ampla variedade de condições (VANCLAY, 1994).

Para o manejo da Floresta com Araucária, manutenção socioambiental e socioeconômica, a con- 
servação e preservação dos ecossistemas florestais requerem a busca de informações que deem suporte à elaboração de planos de manejo com base em modelos gerados utilizando múltiplos fatores relacionados à espécie e ao ambiente. O objetivo deste trabalho foi analisar as relações existentes entre o incremento médio anual em diâmetro e variáveis biométricas, morfométricas e ambientais, como indicativo para uso em modelos de manejo sustentado na região de ocorrência de araucária.

\section{MATERIAL E MÉTODO}

\section{Sítio de estudo}

A área de estudo é uma floresta nativa de Araucaria angustifolia localizada no município de Lages-

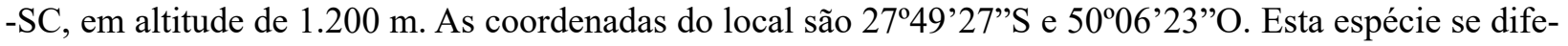
rencia na estrutura adulta da floresta, as copas se encontram na camada superior do dossel e, teoricamente, têm um alto potencial de crescimento.

A região é classificada segundo Köppen como clima $\mathrm{Cfb}$, temperado constantemente úmido, com verão sem estação seca. A temperatura média anual varia de $13,8^{\circ} \mathrm{C}$ a $15,8^{\circ} \mathrm{C}$. A precipitação pluviométrica total anual pode variar de $1.360 \mathrm{~mm}$ a $1.600 \mathrm{~mm}$ e a umidade relativa do ar varia de $80 \%$ a $83 \%$ (EPAGRI, 2002).

$\mathrm{Na}$ área foi realizado inventário, com 35 parcelas permanentes de $400 \mathrm{~m}^{2}$ de área distribuídas de forma sistemática. Todas as árvores com diâmetro a partir de $10 \mathrm{~cm}$ foram plaqueteadas, numeradas e georreferenciadas para uso em medições futuras. Para o estudo de crescimento foram analisadas apenas 26 destas parcelas.

\section{Dados Biométricos e Ambientais}

Nas parcelas amostrais foram medidas as variáveis de diâmetro a 1,30 m de altura do solo (DAP) e altura total, com auxílio de fita métrica e Trupulse. Com estes dados selecionaram-se os indivíduos dominantes (aqueles com maior DAP dentro de cada parcela) e competidores definidos como aqueles em que a copa estava sofrendo influência da árvore dominante. Ainda se obteve a distância entre a árvore dominante e seus competidores, bem como quatro raios de copa, nas direções norte, sul, e longitude leste, oeste, considerando a distância do tronco até o ponto extremo da projeção da copa.

Com as quatro projeções do raio de copa, obteve-se o raio médio de copa $\left(\overline{r c}=\sum_{i=1}^{n=4} \frac{r c}{4}\right)$, o diâmetro de copa $(D c=2 * \overline{r c})$, e, a área de projeção de copa, $\left(A c=\pi * \overline{r c}^{2}\right)$.

Foi realizada a tradagem no tronco de 121 árvores, sendo 26 dominantes e 95 competidoras. Em cada árvore foram retiradas duas amostras, compondo então 242 rolos de incremento. Utilizou-se o trado de Presler de $30 \mathrm{~cm}$ de comprimento e $5 \mathrm{~mm}$ de diâmetro, para obtenção dos rolos e posterior medição do incremento anual em diâmetro. O rolo de incremento foi extraído radialmente na altura do DAP. Os rolos foram retirados perpendicularmente, perfurando até o limite final do trado para obter o maior número de séries temporais do incremento e sempre relativo a uma posição geográfica. Os rolos de incremento foram secos em temperatura ambiente, colados em suporte, lixados e marcados os anéis com auxílio de um microscópio estereoscópio e depois medidos em mesa de mensuração com precisão de $0,01 \mathrm{~mm}$, utilizando o Lintab 6, sendo os dados analisados no Programa Time Series Analysis Program - TSAP-Win da empresa Rinntech ${ }^{\circledR}$.

Os indivíduos foram separados em 3 classes diamétricas, sendo a classe 1(C1) formada com indivíduos que apresentaram DAP de $10 \mathrm{~cm}$ a $29,9 \mathrm{~cm}$, a classe 2(C2) entre 30 e 49,9 $\mathrm{cm}$ e a classe $3(\mathrm{C} 3)$ acima de $50 \mathrm{~cm}$ de DAP.

O banco de dados foi formado por uma matriz com as 121 árvores (linhas) e 23 variáveis do ambiente, dendrométricas e morfométricas (colunas), que incluiu direcionamento de fluxo (DF), acúmulo de fluxo (AF), radiação solar (RS), face de insolação (FI), declividade (DEC), altitude (ALT), incremento médio em diâmetro (IMD), diâmetro (DAP), altura (H), distância (DIST) (dominante/competidor) raios de copa por direção geográfica (NRC1; SRC2; LRC3; ORC4), área basal (G), idade (I), raio médio de copa (RMC), diâmetro de copa (DC), área de copa (AC), incremento em DAP por direção geográfica (Norte, Sul, Les- 
te ou Oeste). As variáveis ambientais descritas consideraram as ferramentas de modelagem hidrológicas. Estas variáveis foram obtidas por meio dos algoritmos descritos por Jenson e Domingue (1988), a partir do Modelo Digital de Elevação (MDE) da área. Estes algoritmos, aplicados para os estudos hidrológicos, permitem analisar variações da superfície da Terra os quais podem ser conectados a modelos ecológicos (THOMPSON; BELL; BUTLER, 2001) e dendrológicos.

Com a composição da planilha, no programa Statistica 7 aplicou-se a análise multivariada com uso das técnicas de análise de Cluster, método de encadeamento Ward's e medida de distância o coeficiente $r$-Pearson, análise fatorial para compreender a inter-relação entre as variáveis e descrever o conjunto de categorias básicas, chamadas fatores, e a análise de componentes principais (ACP) de variáveis para distinguir a variação do peso da contribuição de cada variável (crescimento-ambiente-morfometria) para obter informações e conhecimento para uso em modelos que permitam empregar a complexidade da relação entre as mudanças das variáveis, no incremento diamétrico e condução da estrutura da floresta e seus indivíduos.

\section{RESULTADOS E DISCUSSÃO}

\section{Dados da floresta}

Os dados biométricos das parcelas amostradas demonstraram uma estrutura diamétrica de $\mathrm{J}$ invertido, como observado na Figura 1. No total foram instaladas 35 parcelas, totalizando 424 árvores sendo $8,25 \%$ destas dominantes, $26,89 \%$ competidoras e $64,86 \%$ demais indivíduos alocados dentro das parcelas.

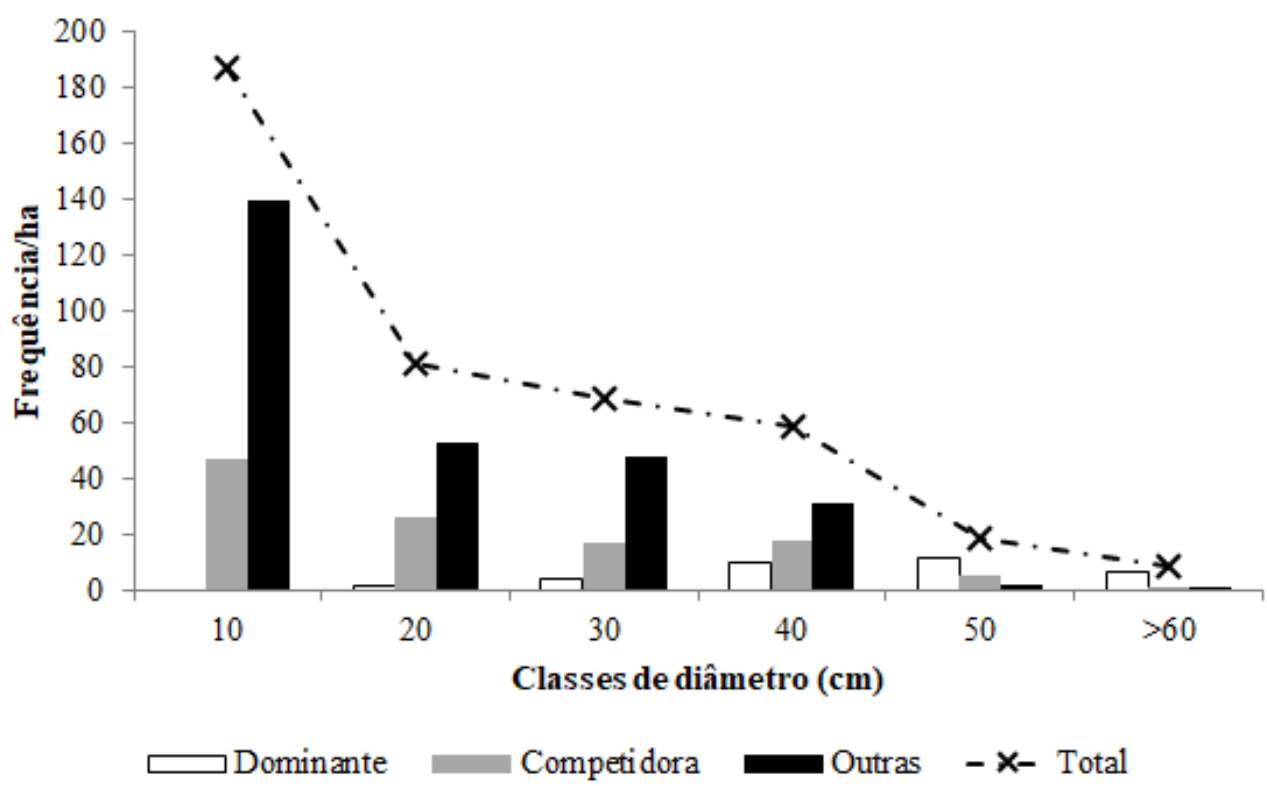

FIGURA 1: Número de árvores e sua distribuição em cada categoria de análise em povoamento natural. FIGURE 1: Number of trees and their distribution in each category of analysis in natural population.

A amplitude dos diâmetros variou de 10 a $87,6 \mathrm{~cm}$ com média de $26,8 \mathrm{~cm}$, enquanto a altura variou de 5,3 a $21 \mathrm{~m}$ com média de 12,9 m. A distribuição diamétrica assemelha-se a um J-invertido caracterizando a estrutura de um povoamento natural (FELFILI, 1997; AUSTREGÉSILO et al., 2004; ALVES JUNIOR et al., 2010; HESS et al., 2010).

Os anéis de crescimento foram contados e, de acordo com as amostras, obteve-se a análise retrospectiva de 13 a 62 anos. O incremento médio anual geral em diâmetro foi de $0,708 \mathrm{~cm}$.ano-1, sendo que, entre as classes, houve diferença no crescimento. A menor classe, $\mathrm{C} 1$, apresentou um crescimento de $0,54 \mathrm{~cm} . \mathrm{nno}^{-1}$, a classe $\mathrm{C} 2$, intermediária, apresentou um crescimento de $0,717 \mathrm{~cm} \cdot a^{-1}$ e a classe $\mathrm{C} 3$ das árvores de maior diâmetro um crescimento de $0,66 \mathrm{~cm}^{-a n o^{-1}}$. Sendo que no total das parcelas, as dominantes apresentaram crescimento de $0,66 \mathrm{~cm} \cdot$ ano $^{-1}$ enquanto as competidoras apresentaram um crescimento de $0,54 \mathrm{~cm} \cdot$ ano $^{-1}$. 


\section{Análise multivariada}

A técnica de análise de agrupamento (Cluster) formou o dendrograma (Figura 2), com seus respectivos grupos e relações, sendo a linha vertical o índice de similaridade e a escala horizontal a ordem de agrupamento dos indivíduos. As linhas verticais partem dos indivíduos e têm altura correspondente ao nível em que os indivíduos são considerados semelhantes.

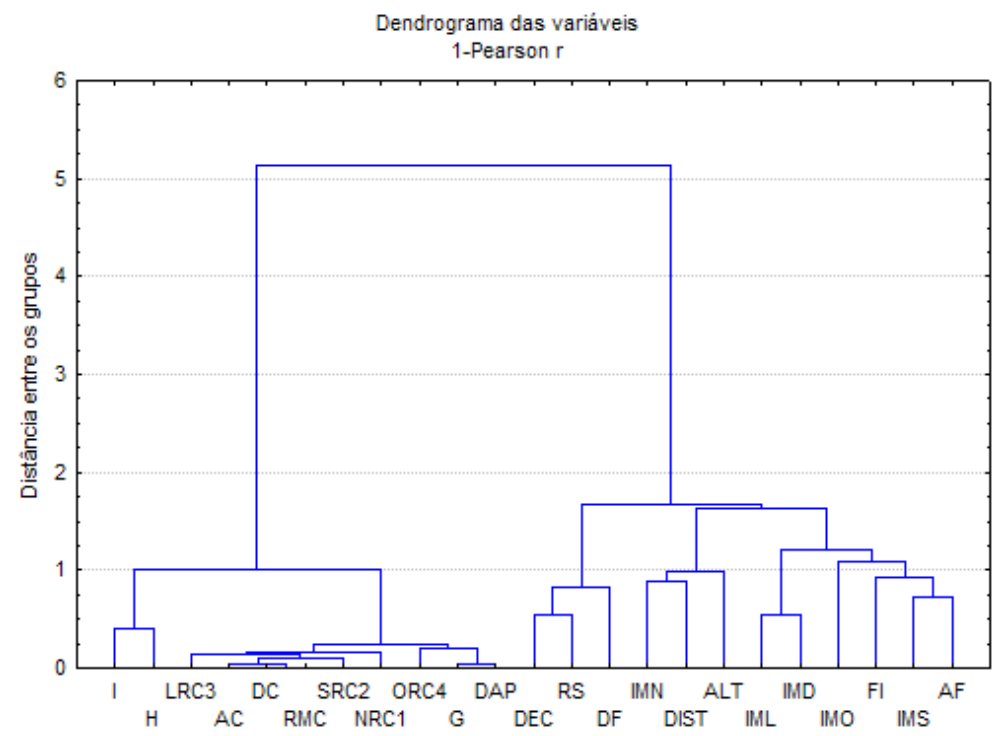

FIGURA 2: Dendrograma das variáveis dendrométricas, morfométricas e ambientais para indivíduos de araucária, sendo: $\mathrm{DF}=$ direcionamento de fluxo; $\mathrm{AF}=$ acúmulo de fluxo; $\mathrm{RS}=$ radiação solar; $\mathrm{FI}=$ face de insolação; $\mathrm{DEC}=$ declividade; $\mathrm{ALT}=$ altitude; IMD = incremento médio em diâmetro; DAP = diâmetro a altura do peito; $\mathrm{H}=$ altura, $\mathrm{DIST}=$ distância (dominante/competidor); $\mathrm{RC}=$ raios de copa nas direções norte, sul, leste e oeste; $\mathrm{G}$ = área basal; $\mathrm{I}=$ idade; $\mathrm{RMC}=$ raio médio de copa; $\mathrm{DC}=$ diâmetro de copa; $\mathrm{AC}=$ área de copa; IMN, IML, IMO, IMS = incremento médio em diâmetro norte, leste, oeste e sul.

FIGURE 2: Dendrogram of dendrometric, morphometric and environmental variables for individuals of araucária, which: $\mathrm{DF}=$ flow direction; $\mathrm{AF}=$ flow accumulation; $\mathrm{RS}=$ solar radiation; $\mathrm{FI}=$ the face of insolation; $\mathrm{DEC}=$ slope ALT $=$ altitude; IMD = average diameter increment; DAP = diameter at breast height (DBH); $\mathrm{H}=$ height; DIST = distance (dominant/competitive); $\mathrm{RC}=$ canopy rays in the north, south, east and west; $\mathrm{G}=$ basal area; $\mathrm{I}=$ age; $\mathrm{RMC}=$ average ray of canopy; $\mathrm{DC}=$ canopy diameter; $\mathrm{AC}=$ canopy area; IMN, IML, IMO, IMS = average increment in diameter north, south, east and west.

Observando a Figura 2, com base na "Linha Fenon" na distância três do eixo vertical, percebe-se a existência de dois grupos homogêneos distintos: o primeiro explicando a relação do incremento médio diamétrico (IMD) com as variáveis ambientais e de competição (distância entre indivíduos). Pode-se notar que a distância (dominante/competidor) mostrou relação com a variável altitude e incremento face norte, e o incremento face sul, relação com acúmulo de fluxo e face de insolação.

Esse grupo demonstra que a relação entre as variáveis do ambiente explica o crescimento da araucária de forma mais representativa, pois apresentam maior altura em relação ao eixo $y$, do que as dimensões e morfometria individual da árvore. Resultados que confirmam a relação das variáveis do ambiente (precipitação, temperatura, teor de umidade, face de exposição) no crescimento também foram observados por Worbes et al. (2003), Zanon e Finger (2010), Moya e Lara (2011) e Cattaneo et al. (2013).

Algumas variáveis do ambiente não podem ser controladas, pois ocorrem independentemente da ação antrópica, ficando o crescimento e a produção atrelados às variáveis que podem ser administradas com intervenções silviculturais que modifiquem condições do ambiente e que favoreçam melhorias no rendimento produtivo. Contudo, as variáveis do ambiente expressam uma relação direta no crescimento, enquanto a competição e a mudança de forma do indivíduo ocorrem somente em conjunto com o crescimento, por 
isso, a maior relação encontrada no dendrograma do incremento com as variáveis ambientais.

A distribuição dos recursos entre as árvores é de difícil mensuração, mas assumindo-se que as taxas de crescimento podem ser proporcionais ou absolutas, a relação entre crescimento-diâmetro é usada como uma proximidade da relação recurso-diâmetro (WICHMANN, 2001; PRETZSCH; BIBER, 2010).

O segundo grupo foi formado pelas variáveis dendrométricas e morfométricas das árvores, as quais estão relacionadas com a estrutura da floresta, pois mostram que a taxa de incremento diamétrico está relacionada ao espaço de crescimento, competição, densidade, o que confirmam Bauhus (2009) e Pretzsch et al. (2014), ao afirmarem que as perturbações naturais (mortalidade, queda de árvores, ataque de insetos, desbastes) abrem espaços no dossel, as quais modificam condições do ambiente (luminosidade, temperatura, recursos disponíveis) seguindo de competição por esse espaço, requisitado pelas árvores e ocupado pela vizinha mais competitiva, ocasionando ainda variações nas taxas de crescimento. Tal condição sugere a formação dos dois grupos e sua relação com o incremento.

A idade mostrou associação direta com as variáveis dendrométricas e morfométricas, porém, como um grupo distinto. A idade como grupo distinto segue uma analogia com base em que nem sempre maiores idades indicam maior crescimento, o que deveria se esperar, contudo, árvores crescendo em espaço reduzido tendem a diminuir a taxa de incremento, necessitando de uma maior idade (muito tempo) para atingir uma maior dimensão.

Fisiologicamente, o incremento ou crescimento não ocorre de forma semelhante em uma mesma idade ou uma mesma dimensão para todos os indivíduos, não podendo esse valor ser usado como referência para um mesmo período de tempo, ou idade. Pode-se observar essa característica com base no valor de incremento para as classes de estudo, pois a classe $\mathrm{C} 1$ de menor diâmetro, consequentemente, menor idade, mas que apresenta um número maior de indivíduos por área, demonstrou menor crescimento devido ao efeito da competição.

Moya e Lara (2011) também salientam que as variações interanuais devido às condições ambientais rigorosas se veem refletidas nos anéis de crescimento das espécies florestais, permitindo estabelecer a variabilidade climática como um ente regulador do crescimento.

$\mathrm{Na}$ ciência florestal, as pesquisas e o desenvolvimento de índices de competição têm sido aplicados, sobretudo, na prognose do crescimento em plantios florestais ou grupos de espécies em florestas naturais (SCHNEIDER; SCHNEIDER, 2008).

Apresentam-se na Tabela 1 os resultados dos autovalores, bem como a porcentagem de variância explicada por cada componente e a variância acumulada pelas mesmas, para servir de subsídio para a análise de Cluster e para corroborar a informação das relações biométricas e ambientais no incremento médio diamétrico.

Como se pode observar, foram necessários seis componentes com autovalores maior que 1 para explicar $81,53 \%$ da variância. Portanto, os dados foram resumidos e somente apresentados para estes componentes principais. Observa-se, ainda, que os dois primeiros componentes possuem autovalores que correspondem a $42,10 \%$ e $9,68 \%$, explicando juntos $51,79 \%$ das variações das medidas originais. 
TABELA 1: Autovalores e percentual da variância explicada de cada componente.

TABLE 1: Eigenvalues and percentage of variance explained for each component.

\begin{tabular}{ccccc}
\hline \multirow{2}{*}{$\begin{array}{c}\text { Número de } \\
\text { componentes }\end{array}$} & \multicolumn{4}{c}{$\begin{array}{c}\text { Autovalores } \\
\text { Extração dos Componentes Principais }\end{array}$} \\
\cline { 2 - 5 } & Autovalor & $\begin{array}{c}\text { Total da variância } \\
\text { explicada em \% }\end{array}$ & $\begin{array}{c}\text { Autovalores } \\
\text { acumulados }\end{array}$ & \% Acumulada \\
\hline 1 & 8,84 & 42,10 & 8,84 & 42,10 \\
\hline 2 & 2,03 & 9,68 & 10,87 & 51,79 \\
\hline 3 & 1,89 & 9,00 & 12,76 & 60,79 \\
\hline 4 & 1,54 & 7,34 & 14,31 & 68,13 \\
\hline 5 & 1,47 & 6,98 & 15,77 & 75,12 \\
\hline 6 & 1,35 & 6,41 & 17,12 & 81,53 \\
\hline
\end{tabular}

A análise de correlação, na análise fatorial demonstrou existir um número representativo de valores superiores $(r=0,7)$ o que significa que a correlação entre as variáveis é de moderada a forte, estando interligadas umas com as outras.

O resultado dos fator loading, com o peso de cada variável que compôs a combinação linear permitiu uma melhor visualização dos fatores em que a proporção de variação das variáveis, está representado na Tabela 2.

TABELA 2: Resultados de fator loading com o peso de cada variável que compôs os seis fatores e que explicam a proporção de variação de cada componente. Foram selecionados os fatores somente com valor $>0,7$.

TABLE 2: Results of fator loading and the weight of each variable composed of the six factors and explaining variation in the proportion of each component. The factors were selected only with value $>0.7$.

\begin{tabular}{c|c|c|c|c|c}
\hline Var./F1 & Var./F2 & Var./F3 & Var./F4 & Var./F5 & Var./F6 \\
\hline DAP - $(0,93)$ & & & & \\
NRC1 - $(0,9)$ & & & & \\
SRC2 - $(0,93)$ & & & & \\
LRC3 - $(0,9)$ & & & & \\
ORC4 - $(0,89)$ & RS - $(0,96)$ & DF - $(0,89)$ & IMN - $(0,94)$ & IMO - $(0,93)$ & IML - $(0,93)$ \\
G - $(0,93)$ & & & & \\
RMC - $(0,99)$ & & & & \\
DC - $(0,99)$ & & & & \\
AC - $(0,98)$ & & & & \\
\hline
\end{tabular}

Em que: Var./F: variável e fator de 1 ao 6, entre parênteses o peso de cada variável; DAP: diâmetro a altura do peito, NRC1, SRC2, LRC3, ORC4: raio médio de copa norte, sul, leste e oeste; G: área basal em m²; RMC: raio médio de copa; DC: diâmetro de copa; AC: área de copa, RS: radiação solar; DF: direção de fluxo; FI: face de insolação, IMN, IMO, IML: incremento médio norte, oeste e leste.

O Fator 1 foi o que apresentou maior número de variáveis e devido à inter-relação, atuam em conjunto para explicar as consequências da densidade no incremento diamétrico, na forma, tamanho de copa e capacidade produtiva da espécie, explicado pela presença de todas variáveis morfométricas no fator. Os demais fatores, 2 a 6, foram representados pelas variáveis ambientais RS, DF, FI e de orientação do incremento, de forma isolada, somente um e dois componentes em cada fator. A análise demonstra que a face de 
exposição pode apresentar diferenças nas médias de incremento e que a face de exposição, quantidade de insolação e o acúmulo de fluxo atuam isoladamente no crescimento, comprovando que modelos do terreno e variáveis dendrocronológicas podem compor modelos de gestão florestal complementando a informação de Thompson, Bell e Butler (2001).

Pode-se observar que o Fator 1 é o que tem maior relação com o incremento diamétrico, pois é derivado do maior autovalor, explicando $42,1 \%$ dessa relação. As variáveis que mais contribuíram neste fator são representadas pelas medidas biométricas e morfométricas, demonstrando a importância do manejo florestal sobre o crescimento, através da adoção de técnicas silviculturais.

Como salienta Roman, Bressan e Durlo (2009), no estágio atual de desenvolvimento do manejo de espécies florestais nativas, o conhecimento da morfometria, das relações morfométricas e da dinâmica das formas das árvores tornam-se imprescindíveis para aprimorar as intervenções silviculturais, de modo especial, quando se deseja tornar efetiva a utilização de espécies da flora nativa em reflorestamentos com interesses econômicos.

Nutto et al. (2001) salientam que a variável diâmetro, comprimento e superfície de copa também estão diretamente relacionados com o crescimento e a produção de matéria seca da árvore, pois, por meio da captação de luz e do processo fotossintético realizado pela copa da árvore, é possível gerar energia química para seu crescimento. O estudo da copa da árvore é uma variável utilizada em modelos de árvore individual e que usa o diâmetro de copa como variável explicativa.

Os Fatores 2, 4, 5 e 6 foram representados por uma única variável, respectivamente, RS, IMN, IMO, IML, o Fator 3 por DF e FI, ou seja, a influência do meio ambiente sobre o crescimento. Ishii (2000) avaliou durante dois anos os ritmos de crescimento de espécies do pantanal e observou que o crescimento é relacionado com a sazonalidade das chuvas, não havendo crescimento no período de secas.

Condições ambientais (temperatura, umidade, acidez do solo, tempestades e outros distúrbios) afetam o crescimento das árvores, e estas, por sua vez, afetam as condições do ambiente (PRETZSCH, 2009). Os componentes em seis fatores, explicam $39,41 \%$ da variância e podem ser interpretados pela influência das variáveis do ambiente no incremento em diâmetro, podendo ser utilizado como índice para o manejo, controle da densidade e sua contribuição na produção total durante o ciclo de corte. A variável com maior escore foi RS (radiação solar), variável que pode ser favorecida ou conduzida no manejo silvicultural do povoamento o que favorece a fotossíntese, o crescimento e a capacidade produtiva das árvores.

Na sequência, em ordem de valor observa-se FI, IMN, IMO, IML e DF. Esses componentes apresentam importância no crescimento, pois a qualidade de sítio de uma área florestal é determinada em virtude da ação e interação de fatores bióticos, climáticos, edáficos e topográficos, influenciados pelas práticas silviculturais e de manejo florestal. Izquierdo et al. (2009) também estudaram a relação entre anéis de crescimento-clima e diferentes cenários de densidade e colocam que compreender essa interação é essencial para a preservação dos ecossistemas e o manejo sustentável, sendo a silvicultura o fator-chave para essa interação e o crescimento das árvores.

Na Figura 3(a) encontra-se o diagrama de ordenação das variáveis originais das seis componentes principais. Pode-se observar que algumas variáveis morfométricas e dendrométricas estão sobrepostas (lado direito do círculo), o que demonstra que estas possuem representatividade semelhante. Pode-se observar ainda, que as variáveis ambientais estão próximas ao círculo unitário, denotando uma forte relação com o incremento em diâmetro, enquanto as variáveis morfométricas estão mais afastadas. 
Projeção das variáveis referentes ao Fator $1 \times$ Fator 2 *Variáveis suplementares

(a)

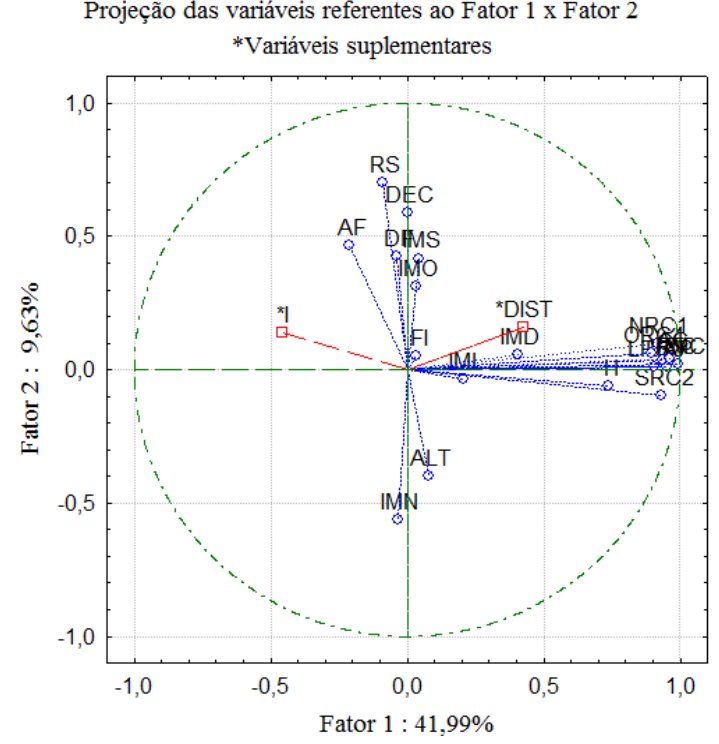

IMD x DIST x Área de copa

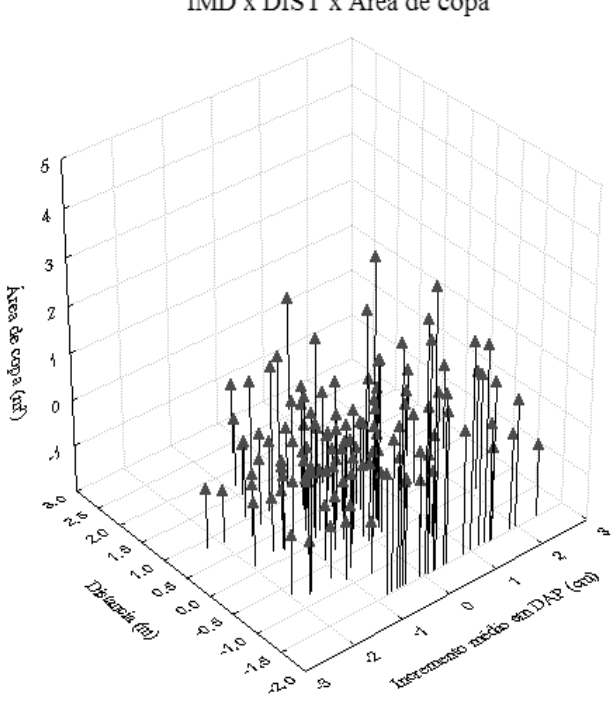

FIGURA 3: Variáveis, suas relações e correlações, em que: (a) círculo das correlações, (b) relação espaço e capacidade de crescimento.

FIGURE 3: Variables, their relationships and correlations, where: (a) relation space and capacity for growth, (b) circle of correlations.

Percebe-se dessa forma (Figura 3b) que, quanto maiores os valores do componente de forma, maiores as dimensões da árvore. Essa componente reúne as características necessárias para ser utilizada no manejo do espaço do sítio, competição, densidade, forma e capacidade de crescimento futuro. Vários autores encontraram relação positiva entre incremento diamétrico e dimensão e forma das árvores, destes citam-se os estudos de Assmann (1970), Hasenauer e Monserud (1996), Monserud e Sterba (1996) e Cunha (2009).

Assim, a forma (copa) e dimensão são componentes importantes para entender o crescimento da árvore e sua relação com o ambiente e estrutura da floresta, sendo seu conhecimento ainda mais relevante em florestas nativas, nas quais diferentes espécies demonstram sua capacidade de aclimatar sua estrutura e competir pelos mesmos recursos e espaço (PRETZSCH, 2009).

$\mathrm{O}$ crescimento depende da quantidade de luz absorvida nas folhas, também da eficiência da conversão de luz absorvida em biomassa e alocação de fotoassimilados nos tecidos (BINKLEY et al., 2013). Em geral, a competição entre árvores tem um efeito negativo na disponibilidade de recursos para árvores individuais, o que se reflete em um declínio no crescimento com um aumento na competição com árvores vizinhas (COATES; CANHAM; LEPAGE, 2009).

A análise multivariada mostrou que existe relação entre forma-dimensão-crescimento de árvores individuais, bem como necessidade de adequação do espaço de crescimento ao longo das mudanças em dinâmica da estrutura horizontal e vertical da floresta, para que as árvores mantenham sua taxa de incremento, crescimento e produção, sendo essas informações aplicáveis para elaboração de planos de manejo e de intervenção silvicultural.

A ACP permite visualizar que em nível de povoamento (PRETZSCH, 2014), entender a variação nas taxas de incremento com as variáveis do ambiente e morfométricas significa a resiliência em produtividade, com redução de espaço descobertos no terreno e, que quanto menor o período de tempo entre a abertura de uma clareira até seu fechamento por árvores vizinhas, maior a utilização de recursos (luz, água, nutrientes) pelos membros do povoamento por tempo e unidade de área.

Das variáveis suplementares, na Figura 3(a), idade (I) não apresentou relação com o incremento médio em diâmetro, nem com as variáveis dendrométricas e morfométricas, pois a mesma se encontra isolada no círculo. Contudo, a distância (DIST) entre os indivíduos apresentou relação com o incremento médio anual em diâmetro, mostrando que existe uma importante relação para o manejo nos quesitos de densidade, uso do espaço, competição, morfometria e dimensões morfométricas. 
A idade (I) apresentou fraca relação biológica com o incremento em diâmetro, o que se explica pelo fato de que as árvores são prioritariamente dependentes dos recursos do sítio, fatores do ambiente, densidade e/ou redução da competição para manter sua taxa de crescimento. A fraca relação biológica da idade demonstra que na floresta existem árvores com mesma dimensão e idades diferentes, bem como árvores de dimensão diferente e mesma idade, pois são os recursos bióticos e abióticos os determinantes do crescimento, a idade não, como ficou demonstrado pela análise multivariada.

\section{CONCLUSÕES}

A análise de Cluster foi útil para separação da grande variedade de dados apresentados, sendo observados dois grupos distintos e seis fatores diferenciados, formados pelas características morfométricas e biométricas e pelas relações do incremento com as variáveis ambientais, que explicaram $81,5 \%$ da variância.

Técnicas de análise multivariada podem ser aplicadas para auxílio na tomada de decisão no manejo florestal. Sua aplicação permitiu correlacionar o incremento médio em diâmetro (que é essencial para compor o ciclo de corte florestal) e sua relação aos fatores do ambiente, índices de forma, espaço e dimensão dos indivíduos.

Embora exista relação do incremento diamétrico com as dimensões e forma das árvores, evidenciou-se a importância dos fatores do ambiente.

\section{REFERÊNCIAS}

ALVES JUNIOR, F. T. et al. Utilização do quociente de Liocourt na avaliação da distribuição diamétrica em fragmentos de floresta ombrófila aberta em Pernambuco. Ciência Florestal, Santa Maria, v. 20, n. 2, p. 307-319, abr./jun. 2010.

ASSMANN, E. The principles of forest yield study. Oxford: Pergamon Press, 1970. 506 p.

AUSTREGÉSILO, S. L. et al. Comparação de métodos de prognose da estrutura diamétrica de uma Floresta Estacional Semidecidual secundária. Revista Árvore, Viçosa, MG, v. 28, n. 2, p. 227-232, 2004.

BAUHUS, J. Rooting patterns of old-growth forests: is aboveground structural and functional diversity mirrowed belowground? In: WIRTH, C. et al. (Ed.). Oldgrowth Forests. Locl: [s. n.], 2009. p. 211-229. (Ecological Studies, 207).

BINKLEY D. et al. Light absorption and use efficiency in forests: why patterns differ for trees and stands. Forest Ecology and Management, Amsterdam, v. 288, p. 5-13, 2013.

CATTANEO, N. et al. Sex-related, growth-climate association of Araucaria angustifolia in the neotropical ombrophilous woodlands of Argentina. Dendrochronologia, Italy, v. 31, p.147-152, 2013. http://dx.doi.org/10.1016/j.dendro.2013.01.005

COATES, K. D.; CANHAM, C. D.; LEPAGE, P. T. Above versus below ground competitive effects and responses of a guild of temperate tree species. Journal of Ecology, Oxford, v. 97, n. 1, p. 118-30, 2009.

CUNHA, T. A. Modelagem do incremento de árvores individuais de Cedrela odorata L. na Floresta Amazônica. 2009. 87 f. Dissertação (Mestrado em Engenharia Florestal) - Universidade Federal de Santa Maria, Santa Maria, 2009.

EPAGRI - EMPRESA DE PESQUISA AGROPECUÁRIA E EXTENSÃO RURAL DE SANTA CATARINA. Dados e informações biofísicas da Unidade de Planejamento Regional Planalto Sul Catarinense - UPR 3. Florianópolis: EPAGRI; CIRAM, 2002. 70 p.

FELFILI, J. M. Diameter and height distributions in a gallery forest community and some of its main species in central Brazil over a six-year period (1985-1991). Revista Brasileira de Botânica, São Paulo, v. 20, p. 155-162, 1997.

FINGER, C. A. G. Biometria florestal. Santa Maria: CEPEF; FATEC; UFSM, 2006. 284 p.

HASENAUER, H.; MONSERUD, R. A. A crown ratio model for Austrian forests. Forest Ecology and Manegement, Amsterdam, v. 84, p. 49-60, 1996.

HESS, A. F. et al. Proposta de manejo de Araucaria angustifolia utilizando o quociente de Liocourt e análise de incremento, em propriedade rural no município de Lages, SC. Pesquisa Florestal Brasileira, 
Colombo, v. 30, n. 64, p. 337-345, nov./dez. 2010.

IBGE. Manual técnico da vegetação brasileira. Rio de Janeiro: IBGE, 1992. 92 p. (Série Manuais Técnicos em Geociências, 1).

ISHII, I. H. Estudos dendrocronológicos em matas ciliares do pantanal. In: COLETÂNEA DE SEMINÁRIOS TÉCNICOS DO CPA-PANTANAL, 1996/1997, Corumbá. Anais... Corumbá: Embrapa Pantanal, 2000. p. 43-44.

IZQUIERDO, G. G. et al. Climate-growth variability in Quercus ilex L. west Iberian open woodlands of different stand density. Annals of Forest Science, Les Ulis, v. 66, p. 1-12, 2009. doi: 10.1051/forest/2009080 JENSON, S. K. E.; DOMINGUE, J. O. Extracting topographic structure from digital elevation data for geographic information system analysis. Photogrammetric Engineering and Remote Sensing, Maryland, v. 54, n. 11, p. 1593-1600, 1988.

LEITE, P. F.; KLEIN, R. M. Vegetação. In: IBGE. Diretoria de Geociências. Geografia do Brasil: Região Sul. Rio de Janeiro: IBGE, 1990. 420 p.

MOYA, J.; LARA, A. Cronologías de ancho de anillos de queñoa (Polylepis tarapacana) para los últimos 500 años en el Altiplano de la región de Arica y Parinacota, Chile. Bosque, Chile, v. 32, n. 2, p. 165-173, 2011.

MONSERUD, R.; STERBA, H. A. A basal area increment model for individual trees growing in even-and-uneven-aged forest stands in Austria. Forest Ecology and Management, Amsterdam, v. 80, p. 57-80, 1996.

NUTTO, L. et al. Utilização dos parâmetros da copa para avaliar o espaço vital em povoamentos de Pinus elliottii Engelm. Boletim de Pesquisa Florestal, Colombo, n. 42, p. 123-138, 2001.

PAULA, A. et al. Sucessão ecológica da vegetação arbórea em uma floresta estacional semidecidual, Viçosa, MG, Brasil. Acta Botânica Brasílica, São Paulo, n. 18, v. 3, p. 407-423, 2004.

PRETZSCH, H. Forest dynamics, growth and yield. Berlin: Springer-Verlag, 2009. 671 p.

PRETZSCH, H. Canopy space filling and tree crown morphology in mixed-species stands compared with monocultures. Forest Ecology and Management, Amsterdam, p. 8-14, 2014. http://dx.doi.org/10.1016/j.foreco.2014.04.027

PRETZSCH, H.; BIBER, P. Size-symmetric versus size-asymmetric competition and growth partitioning among trees in forest stands along an ecological gradient in central Europe. Canadian Journal of Forest Research, Ottawa, v. 40, n. 2, p. 370-384, 2010.

PRETZSCH, H. et al. Effect of variable retention cutting on the relationship between growth of coarse roots and stem of Picea mariana. Scandinavian Journal of Forest Research, Ottawa, p. 222-233, 2014. http://dx.doi.org/10.1080/02827581.2014.903992.

ROMAN, M.; BRESSAN, D. A.; DURLO, M. A. Variáveis morfométricas e relações interdimensionais para Cordia trichotoma (Vell.) Arráb. ex Steud. Ciência Florestal, Santa Maria, v. 19, n. 4, p. 473-480, out./dez. 2009.

SANQUETTA, C. R. et al. Inventários florestais: planejamento e execução. 2. ed. Curitiba: Multi-Graphic, 2009. 316 p.

SCHNEIDER, P. R.; SCHNEIDER, P. S. P. Introdução ao manejo florestal. Santa Maria: FACOS; UFSM, 2008. $566 \mathrm{p}$.

SIMINSKI, A. et al. Sucessão Florestal Secundária no município de São Pedro de Alcântara, litoral de Santa Catarina: estrutura e diversidade. Ciência Florestal, Santa Maria, v. 14, n. 1, p. 21-33, 2004.

SPEER, J. H. Fundamentals of tree-ring research. Tucson: The University of Arizona Press, 2010. 333 p. THOMPSON, J. A.; BELL, J. C.; BUTLER, C. A. Digital elevation model resolution: effects on terrain attribute calculation and quantitative soil-landscape modeling. Geoderma, v. 100, n. 1/2, p. 67-89, mar., 2001. Doi: 10.1016/S0016-7061(00)00081-1.

VANCLAY, J. K. Modelling Forest growth and yield: applications to mixed tropical forests. Wallingford: CAB International, 1994. 312 p.

VELOSO, H. P.; GÓES FILHO, L. Fitogeografia brasileira: classificação fisionômica-ecológica da vegetação neotropical. Rio de Janeiro: IBGE, 1982. 80 p. (Boletim Técnico Projeto Radambrasil, Série Vegetação, 1).

WICHMANN, L. Annual variations in competition symmetry in even-aged Sitka Spruce. 
Annals of Botany, Oxford, v. 88, p. 145-151, 2001.

WORBES, M. et al. Tree ring analysis reveals age structure, dynamics and wood production of a natural forest stand in Cameroon. Forest Ecology and Management, Amsterdam, v. 173, p. 105-123, 2003. doi: 10.1016/S0378-1127(01)00814-3

ZANON, M. L. B.; FINGER, C. A. G. Relação de variáveis meteorológicas com o crescimento das árvores de Araucaria angustifolia (bertol.) kuntze em povoamentos implantados. Ciência Florestal, Santa Maria, v. 20, n. 3, p. 467-476, jul./set. 2010. 